\title{
Sorption Hysteresis of Hardened Cement Pastes
}

\author{
Peter Schiller1, Mirco Wahab'1, Thomas Bier², Sandra Waida'2, Hans-Jörg Mögel1 \\ ${ }^{1}$ Inst. Phys. Chem., TU-Bergakademie Freiberg, Freiberg, Germany \\ ${ }^{2}$ IKGB, TU-Bergakademie Freiberg, Freiberg, Germany \\ Email: mirco.wahab@chemie.tu-freiberg.de
}

Received 9 December 2015; accepted 1 February 2016; published 6 February 2016

Copyright (C) 2016 by authors and Scientific Research Publishing Inc.

This work is licensed under the Creative Commons Attribution International License (CC BY). http://creativecommons.org/licenses/by/4.0/

c) (i) Open Access

\begin{abstract}
Mesopores in porous solids can produce a pronounced sorption hysteresis at moderate and high reduced vapor pressures of the ambient gas that is condensed in the pores. Unlike to other conventional porous materials, cement pastes often behave exceptionally. The water sorption hysteresis frequently persists at very low humidity. This hysteresis is reflected in a corresponding hysteresis loop of the solid skeleton volume. We discuss a theoretical model based on the strong compression force exerted by a condensate on the walls of narrow slit pores embedded in an elastic solid. This compression force is shown to be capable of shifting walls of narrow slit pores. Humidity-dependent closing and reopening of slit pores can produce hysteresis loops even at low humidity.
\end{abstract}

\section{Keywords}

Porous Solids, Sorption Hysteresis, Capillary Forces, Cement-Based Materials

\section{Introduction}

Hardening of cement pastes results from a hydration reaction that forms the C-S-H gel, where the notation $\mathrm{C}=$ $\mathrm{CaO}, \mathrm{S}=\mathrm{SiO}_{2}$, and $\mathrm{H}=\mathrm{H}_{2} \mathrm{O}$ is used [1]. This calcium-silicate-hydrate gel glues together reaction products and non-reacting components within the cement paste. The C-S-H gel is supposed to have a layered structure comparable to the mineral tobermorite. Narrow slit pores in the layered structure can be analyzed by different methods such as X-ray diffraction, neutron scattering and NMR [1] [2]. It was found that slit pores in cement pastes can be rather small, their width may range between a few angstroms and a few nanometers. Micropores, which have a pore width smaller than two nanometers, often comprise a large fraction of the total pore volume in cement pastes. As the C-S-H gel is hydrophilic, the porous material adsorbs water from the atmosphere. Unlike 
many other hydrophilic porous materials [3], the hysteresis of water sorption isotherms in cement-based materials often persists at very low humidity [4].

A comparison of different cement pastes with low and high volume fractions of small pores suggests that sorption hysteresis at low humidity only appears in materials with a large amount of small pores. The diagrams in Figure 1 show a comparison of sorption isotherms for hardened cement pastes with a low (left column) and a high (right column) fraction of micropores. Both pastes have the same chemical composition. Sorption hysteresis loops are reflected in corresponding loops in diagrams for the volume change of the solid skeleton [4]. Micromechanical models for describing the unusual sorption hysteresis should explain this observation.

Several arrangements of the C-S-H sheets in cement pastes have been proposed. Feldman and Serena [5] assumed a porous gel of randomly distributed stacks of C-S-H layers. Jennings [6] proposed a model of colloidal disklike building blocks called globules, which stick together and form a fractal network with small and larger pores. Each globule consists of a stack of parallel C-S-H layers. The sequence of C-S-H layers contains some
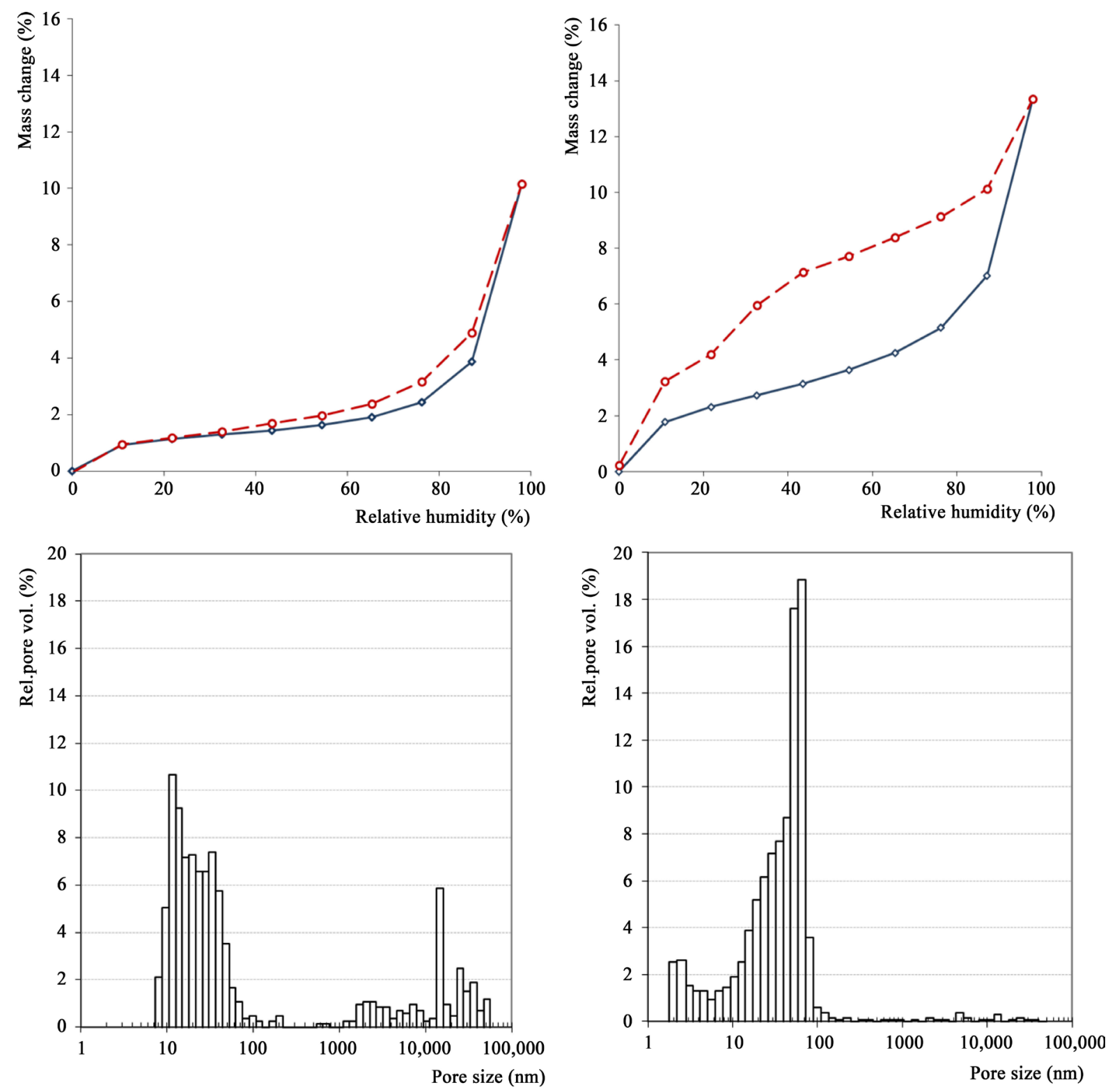

Figure 1. Water sorption isotherms and corresponding pore size distributions (mercury intrusion porosimetry) for Portland cement pastes (CEM-I). The material with a large amount of micropores and small mesopores (right) displays a strong sorption hysteresis in comparison to an $8 \mathrm{~h}$ autoclave-hardened material (left), which has a low fraction of small pores. 
gaps filled with one or a few layers of physically bound water [1]. Such a water layer, which has a width of a few angstroms, could be considered as the condensate in a narrow slit pore located in the space between two adjacent hydrated calcium silicate sheets. Experiments and theoretical reasoning demonstrate that narrow water filled gaps between adjacent C-S-H layers are held together by strong cohesion forces [7] [8].

Conventional capillary forces [9], which are very important for the macroscopic description of the forces in broad water-filled slit pores, play a minor role in micropores of cement pastes. In narrow water-filled slit pores of hardened cement pastes mainly calcium ions cause the main contribution to the negative tension that attracts the pore walls. Thus, the compression force per unit area can be considered as a tension (negative pressure) that the liquid condensate exerts on the pore walls of the slit pore. Since the tension on the walls in hardened cement pastes is much larger than typical capillary forces in many other materials, the planar pore walls may be shifted considerably, although the Young modulus is relatively high [10]. It seems even possible that the compression pressure completely closes narrow slit pores. Closing a pore embedded in an elastic solid requires the expenditure of elastic energy. A closed slit pore can reopen if the wall pressure is diminished after changing external conditions such as relative air humidity. In a previous paper [11] we have shown that closing and reopening of slit pores in soft hydrophilic solids is possible by varying the capillary force, which depends on the air humidity in the environment. This mechanism can produce hysteresis if the elastic energy required to close a pore by a capillary compression force is rather low. The theoretical approach is applicable to soft solids with Young modulus not higher than about $1 \mathrm{GPa}$.

On the other hand, for reopening the pore, the elastic energy must be sufficiently high to overcome the interface energy at the solid-solid contact region of a closed slit pore. It can be shown that sorption hysteresis can appear even at low air humidity, if material parameters satisfy some conditions necessary for closing and reopening the slit pores. If these conditions are satisfied, the resulting sorption hysteresis is also reflected in a hysteresis of the volume change of the solid skeleton. In this paper we consider the possibility of sorption hysteresis resulting from closing and reopening of narrow slit pores in solid materials with relatively high Young modulus such as hardened cement pastes. Apart from the Young modulus, the tension exerted on pore walls is also high in hardened cement pastes. It will be shown that pore walls in cement pastes can be shifted in a similar way as in soft solids. We elucidate conditions for the appearance of low-humidity sorption hysteresis in cement-based materials.

\section{Elastic Energy of the Solid Skeleton Associated with Closed and Partially Closed Slit Pore}

Figure 2 illustrates a slit pore with width $b$ embedded in an elastic medium. If the pore is empty, i.e. if it contains no condensed water, the pore does not exert any stress on the solid medium and the elastic energy is equal to zero. A slit pore can be closed after exerting a compression force that pushes both pore walls together so that the gap between them disappears. Starting from a deformation-free slit pore, the elastic energy required to produce the closed pore configuration can be obtained from the theory of dislocations. In this theory, the shift $b$ (Burgers vector in a crystal lattice) is produced after removing a lattice half plane in a crystal and gluing both sites of the resulting gap together. Hence, the Burgers vector $b$ in crystal physics is always equal to a multiple of a lattice constant.

However, as the elasticity theory is a macroscopic theory, and the solid is considered as a continuous medium without periodic lattice structure, the expression for the elastic energy of a closed pore configuration is also correct when $b$ is not equal to a lattice constant. Thus, the elastic energy of the closed pore shown in Figure 2(b) can be evaluated by applying the same expression as used for evaluating the elastic energy of an edge dislocation. The elastic energy is concentrated along the dislocation line, which is the rim of the closed pore (direction of the $y$-axis in Figure 2(b)). A small cylindrical region around the dislocation line (core radius $r_{0}$ ), where elastic stresses diverge, is excluded, since the linear elasticity theory is not applicable there. Let $E$ and $v$ be the Young modulus and Poisson's ratio, respectively. Then the elastic energy per dislocation line length $L$ (length of the slit pore along the $y$-axis) embedded in a solid cylinder with radius $R$ is [12]

$$
\frac{W_{e l}}{L}=\frac{E b^{2}}{8 \pi\left(1-v^{2}\right)} \ln \left(\frac{R}{r_{0}}\right)
$$

The core radius $r_{0}$ is generally assumed to be comparable with a lattice constant $\left(r_{0} \simeq 0.3 \mathrm{~nm}\right)$. Unfortunately, 


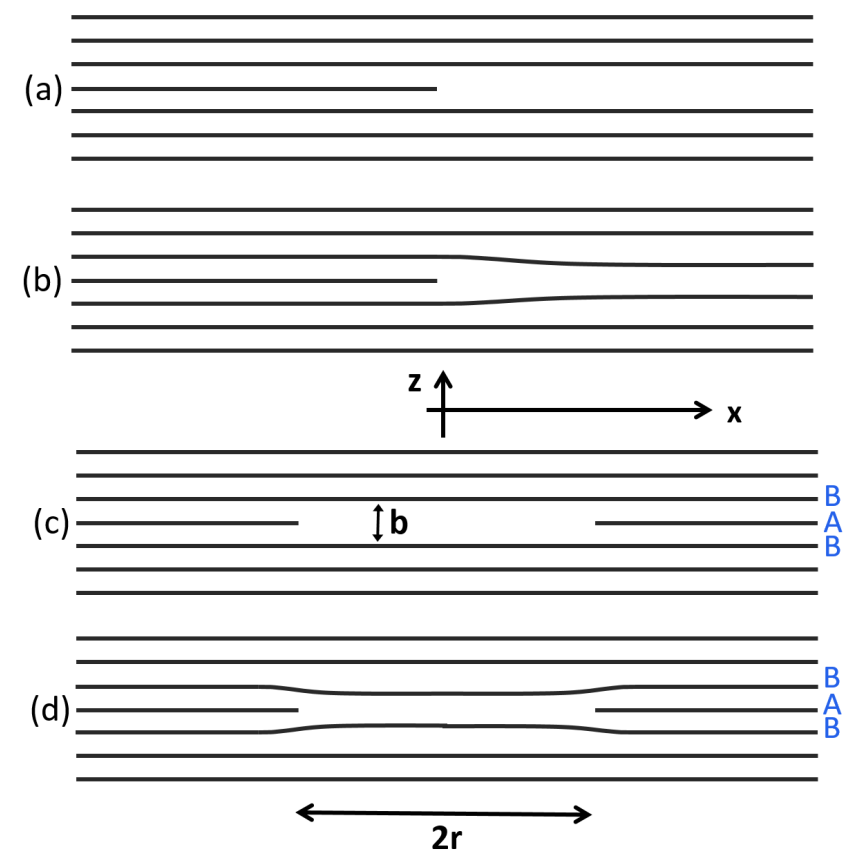

Figure 2. (a) A lattice half-plane of a layered crystal lattice is removed; (b) The edge dislocation resulting after gluing both opposite solid surfaces together can be considered as a closed slit pore. (c) In a crystal lattice with A and B layers an open circular slit pore with radius $r$ and thickness $H_{0}=b$ is formed by removing the atoms in a circular region of an atomic A-plane. (d) A dislocation appears if both opposite interfaces (B-planes) in the pore are glued together (after [13]). The circular dislocation line, which is considered as the borderline of the closed slit pore, is located in the $x-y$ plane and the Burgers vector $\mathrm{b}=\left(0,0, b=H_{0}\right)$ is parallel to the $\mathrm{z}$-axis of the Cartesian coordinate system.

$W_{\text {el }}$ (Equation (1)) increases logarithmically with increasing radius of a cylindrically shaped elastic solid. To avoid this divergence, we consider a circular slit pore with radius $r$ and thickness $b=H_{0}$. The corresponding model in solid state physics is a closed circular dislocation line. Thus, using an approximate expression known from dislocation theory, the elastic energy required to close the pore is [13]

$$
W_{e l}=\frac{E b^{2} r}{4\left(1-v^{2}\right)} \ln \left(\frac{2 r}{r_{0}}\right)
$$

This energy may be compared with the adhesion energy of the solid-solid contact $W_{s}=\pi r^{2} w$, which is required to reopen the slit. $w$ can be considered as the adhesion energy per unit area for two planar solid surfaces immersed in water.

We expect that the slit pore is open if $W_{\mathrm{el}}>W_{S}$, and completely closed if $W_{S}>W_{\mathrm{el}}$. Hence, equation $W_{\mathrm{el}}=W_{S}$ defines a threshold value $b_{0}$ at which the pore is completely closing. Instead of $b_{0}$, we can also evaluate a threshold value $E_{0}$ for Youngs's modulus

$$
E_{0}=\frac{4 \pi\left(1-v^{2}\right) r w}{b^{2} \ln \left(2 r / r_{0}\right)}
$$

where $b=H_{0}$ is the width of the open pore without condensed water. If $E<E_{0}$ the closed pore is stable, whereas for $E>E_{0}$ this pore configuration is unstable. In the case $w=0.1 \mathrm{~J} / \mathrm{m}^{2}, r=10 \mathrm{~nm}, b=1 \mathrm{~nm}, \mathrm{v}=0.3$ and $r_{0}=0.3$ $\mathrm{nm}$, we obtain $E_{0}=40 \mathrm{GPa}$. If we assume that $w$ results from van-der-Waals interactions, the value of $w$ may be obtained from the Hamaker constant. In this case, $w$ is estimated to vary in the range between $0.005 \mathrm{~J} / \mathrm{m}^{2}$ and $0.01 \mathrm{~J} / \mathrm{m}^{2}$ [14], and then the threshold $E_{0}$ is supposed to be much lower $\left(E_{0} \simeq 2 \mathrm{GPa}\right)$. Closing and reopening of narrow slit pores in hardened cement pastes could be driven by the dependence of Young's modulus $E$ on air humidity. The elastic modulus of Portland cement at 100\% relative air humidity was found to be about twice times larger than its value at relative humidity smaller than $20 \%$. Hence, narrow slit pores with appropriate aspect ratios $b / 2 r$ could be open at high air humidity and closed at low air humidity. 
Equation (2) can be generalized to the case of a slit pore that contains one or a few water layers. Micropores with pore wall distances $H$ lower than one nanometer are ubiquitous in cement-based materials. Let $H_{0}$ be the width of the force-free open slit pore which contains no condensed water. The wall distance adapts to the layered water which produces a compression force. When a pore is not completely closing and the slit still contains one or a few water layers, the wall shift $b$ is smaller than $H_{0}$. Thus, inserting $b=H_{0}-H$ into Equation (2), we obtain the elastic energy for the partially compressed circular slit pore

$$
W_{e l}=\frac{E\left(H_{0}-H\right)^{2} r}{4\left(1-v^{2}\right)} \ln \left(\frac{2 r}{r_{0}}\right)
$$

\section{Sorption Hysteresis}

The evaluation of the threshold value of Young's modulus $E_{0}$ (Equation (3)) for closing and reopening a slit pore neglects the tension of condensates on pore walls. In a previous paper [11] we have demonstrated how closing and reopening of slit pores in soft solids can produce sorption hysteresis, when capillary forces of the condensate are taken into account. Conventional capillary forces, which are supposed to be the driving force for the shifting walls of slit pores, are sufficient to close narrow slit pores in soft solids. In more rigid materials such as hardened cement pastes the capillary pressure obtained from a macroscopic description is too weak to close pores.

However, Monte-Carlo simulations suggest that the tension exerted by adsorbates on the walls of micropores could be much larger than predicted by the classical theory of capillarity. A theoretical description of the wall tension for slit pores can start from the grand potential $\Omega$ that includes contributions resulting from the interaction between adsorbate molecules and substrate-adsorbate interactions. In the present case, where the pore can change its width, we add the elastic energy contribution $W_{\mathrm{el}}$ produced by the deformation of the solid skeleton. Let us first consider a closed circular pore as a reference state. According to Equation (4), the elastic energy per unit area is

$$
\frac{\Omega_{\text {closed }}}{\pi r^{2}}=\frac{1}{2} k H_{0}^{2}
$$

where the factor $k$ is defined as

$$
k=\frac{E}{2 \pi r\left(1-v^{2}\right)} \ln \left(\frac{2 r}{r_{0}}\right) .
$$

If the pore opens and the distance between the walls increases, work must be done to overcome the attractive forces between the pore walls. The work per unit area $w(H)$ may be evaluated by Monte-Carlo simulations of a grand canonical ensemble [8]. Thus, the grand potential per unit area is written as

$$
\frac{\Omega_{\text {open }}(H)}{\pi r^{2}}=w(H)+\frac{1}{2} k\left(H_{0}-H\right)^{2}
$$

Mechanical stability of the open pore configuration requires that $\partial \Omega_{\text {open }}(H) / \partial H=0$. Thus, we obtain an equation for the reduced pore width

$$
\frac{H}{H_{0}}=1+\frac{p(H)}{p_{0}}
$$

where $p_{0}=H_{0} k$ is a constant and

$$
p(H)=-\frac{\partial w(H)}{\partial H}
$$

is the tension of the condensate exerted on the pore walls. Obviously, the slit pore is completely closed if $p(H) \leq$ $-p_{0}$. Let us assume that the pore is partially open. The condition for a minimum of the grand potential necessary for the stability of the open pore, $\partial^{2} \Omega_{\text {open }}(H) / \partial H^{2}>0$, yields

$$
\frac{\partial p(H)}{\partial H}<k \text {. }
$$


If more than one minimum of $\Omega_{\text {open }}(H)$ exists, the value of $H$ with the lowest thermodynamic potential $\Omega_{\text {open }}(H)$ corresponds to the absolutely stable pore configuration. However, hysteresis is only possible when the system can remain trapped in a metastable state, which corresponds to a minimum but not the absolute minimum of the thermodynamic potential $\Omega(H)$.

Let us first discuss the mechanism of pore closing and reopening in the framework of the conventional macroscopic theory of capillarity. This macroscopic theory could be applied to porous soft solids. In a previous paper we have shown that empty slit pores embedded in a soft elastic solid are not stable if the wall distance $H_{0}$ is lower than a critical value [11]. Thus, narrow slit pores are either filled with condensate or they are closed $(H$ $=0$ ). Here, we assume that $H_{0}$ is sufficiently small, and the unstable empty state of the pore can be disregarded.

\subsection{Sorption and Deformation Hysteresis for Slit Pores Embedded in Soft Solids}

Broad slits of soft solids can be closed by the compression force exerted by a condensate. In this case, the macroscopic classical theory of capillarity is appropriate to describe hysteresis. Starting from Equation (7), the contribution $w(H)$ to the grand potential is [9]

$$
w(H)=w-p H
$$

where

$$
p=\rho k T \ln (m)
$$

is the condensate pressure. Here, $\rho$ denotes the number density of the condensate, $k$ the Boltzmann constant, $T$ the temperature and $m$ the relative humidity of the water vapor, which is in thermodynamic equilibrium with the condensate in the slit pore. Obviously, as long as the pore is completely filled with condensate, the condensate pressure $p$ is independent of $H$, i.e. we have $p(H) \equiv p$. Using Equations (5) and (7), we define a scaled thermodynamic potential $\omega(H)=\left(\Omega_{\text {open }}(H)-\Omega_{\text {closed }}\right) / \pi r^{2} w$. Furthermore, after replacing $H$ in $\omega(H)$ by $H=H_{0}\left(1+p / p_{0}\right)$ (Equation (8)), we arrive at

$$
\omega(p)=1-K\left(1+\frac{p}{p_{0}}\right)^{2}
$$

where $K=k H_{0}^{2} / 2 w$ is a dimensionless parameter. Taking into account that $p_{0}=k H_{0}$, this parameter can also be written as

$$
K=\frac{H_{0} p_{0}}{2 w} .
$$

In Figure 3 the reduced pore wall thickness $H / H_{0}$ is plotted versus the reduced condensate tension $p / p_{0}$. The pore is open and filled with condensate if $\omega(p)<0$ (line $a \rightarrow d$ ), and along the line $d \rightarrow b$ (metastable states). Completely closing and partially reopening of a pore occurs if $K>1$, and if the reduced condensate tension $p / p_{0} \propto \ln (m)$ varies in the range

$$
-1<\frac{p}{p_{0}}<-1+\frac{1}{\sqrt{K}}
$$

For considering the hysteresis in more detail, it is useful to introduce the notation $\Pi=p / p_{0}$ for the scaled condensate tension $(\Pi \leq 0)$. At $\Pi_{c}=-1+1 / \sqrt{K}$ a closed pore must reopen, and for $\Pi>\Pi_{c}$ the open pore configuration is absolutely stable $(\omega(p)<0)$. Figure 3 illustrates the hysteresis loop accompanied with pore closing and reopening. At a starting point $\Pi \geq \Pi_{d}$, when the conditions $K>1$ and $-1+1 / \sqrt{K}<\Pi<0$ are satisfied, the pore is open. Increasing the magnitude of pore pressure $\Pi$ along the path $a \rightarrow b$ the reduced pore thickness gradually decreases to zero (Equation (8)). The condition of mechanical stability (Equation (10)) is always satisfied. At point $\mathrm{b}$, where $H$ is equal to zero and the pore is closed, the contribution of the surface energy term $w$ (first constant term 1 in Equation (13)) disappears in the thermodynamic potential $\omega(p)$, and $\omega(p)$ switches from 1 (point b) to zero (point e in Figure 3). After arriving at the closed configuration e, the 


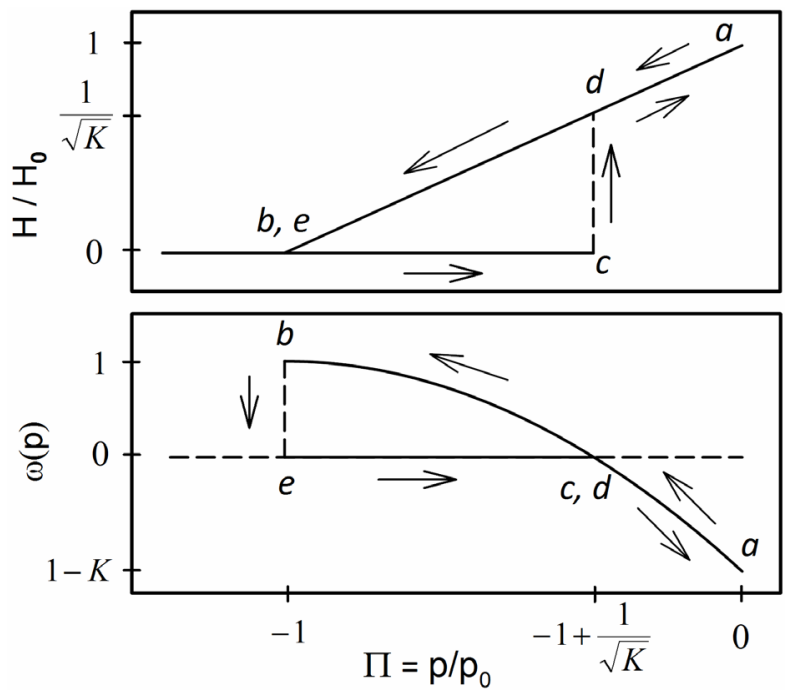

Figure 3. Reduced pore wall distance $H / H_{0}$ and thermodynamic potential $\omega(p)$ versus the reduced wall tension $\Pi=p / p_{0}$. The arrows indicate the path of the hysteresis loop.

pore remains closed even after a slight increase of $\Pi$. If the pore partially reopened, the reduction of elastic energy would be lower than the expenditure of surface energy $w$. Thus, the pore remains closed along the line $e$ $\rightarrow c$. However, if $\Pi$ is larger than $\Pi_{c}=\Pi_{d}=-1+1 / \sqrt{K}$, the thermodynamic potential difference $\omega(p)$ becomes negative and the partially open pore has a lower grand potential than the closed one.

Thus, the reduced pore width $H / H_{0}$ switches from zero to $1 / \sqrt{K}$, which corresponds to the transition $c \rightarrow d$ in Figure 3. The path along the hysteresis loop $d \rightarrow b \rightarrow e \rightarrow c \rightarrow d$ can be repeated without causing any damage in the material, since only elastic deformations of the solid occur.

\subsection{Sorption and Deformation Hysteresis in the Case of More Rigid Solids}

Equation (11) for potential $w(H)$ provides us only a crude macroscopic description. Broad pores embedded in rigid materials with Young's modulus larger than a few GPa do not close when the relatively weak pore tension evaluated by Equation (12) is exerted. In rigid solids such as hardened cement pastes broad slits with wall distances exceeding a nanometer cannot close. The evaluation of the strong condensate tension $p(H)$ in narrow pores requires the application of a microscopic theory. A recent neutron scattering study confirmed that the distance $H$ between hydrated calcium silica layers of the lamellar C-S-H structure increases with increasing water content [1]. This observation suggests that the tension $p(H)$ between C-S-H layers should depend on the relative humidity $(m)$ of the atmosphere. Using Monte-Carlo simulations for a grand canonical ensemble, Bonnaud et al. [8] obtained some numerical data for the tension $p(H)$ exerted on the pore walls of slits by layers of water in combination with divalent cations in a C-S-H phase.

Unfortunately, in [8] only one curve that shows the dependence of the tension $p(H)$ on $H$ in the range $0 \leq H \leq 1 \mathrm{~nm}$ is published. This curve refers to the case of maximal relative humidity $(m=1)$. The values of $p(H)$ are found to be monotonously increasing in the range between $-20 \mathrm{GPa}$ at $H=0.1 \mathrm{~nm}$ and $-3 \mathrm{GPa}$ at $H=1$ $\mathrm{nm}$. A sketch, which shows roughly the qualitative features of the function $p(H)$, is illustrated in Figure 4 (curve a). Let us assume that at the pore wall distances $H=H_{1}$ and $H=H_{2}$ the slopes $\partial p(H) / \partial H$ of the depicted tangents are equal to $k$. Then, taking into account the stability condition (Equation 10), the slit pore should be stable for $H<H_{1}$ and $H>H_{2}$. A pore width in the range $H_{1}<H<H_{2}$ is mechanically unstable $(\partial p(H) / \partial H>k)$. Pores with wall distances in the unstable region cannot exist. When the limits $H=H_{1}$ and $H$ $=H_{2}$ of stable pore width regions are crossed, the pore wall distance $H$ must jump towards a stable state. Usually, discontinuous transitions are accompanied with hysteresis. Thus, we expect hysteresis if the pore tension $p(H)$ varies and crosses the stability limits $p\left(H_{1}\right)$ and $p\left(H_{2}\right)$. A complete elucidation of the hysteresis behavior of a pore requires a detailed knowledge of the function $p(H)$ in dependence on relative humidity $m$. Let us estimate the range of the hysteresis region for the condensate tension data $p(H)$ simulated by Bonnaud et al. [8] for a pore width $H_{0}=1 \mathrm{~nm}$. Assuming reasonable values for the Young modulus $(E=40 \mathrm{GPa})$, aspect ratio of the slit 


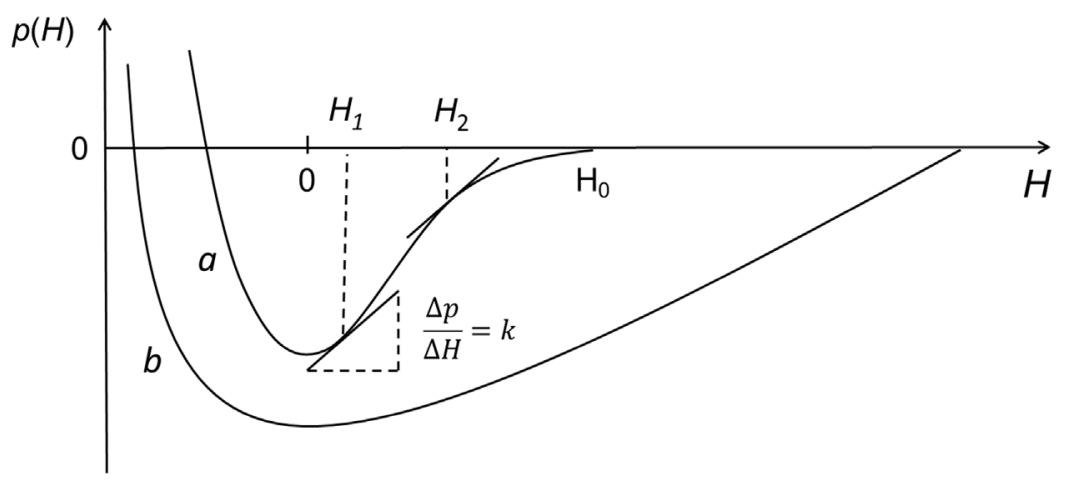

Figure 4. Condensate tension $p(H)$ versus the wall distance $H$ of slit pores. In case $a$ the slit pore is mechanically unstable in the range $H_{1}<H<H_{2}$. Curve $b$ shows a possible function $p(H)$ for a slit pore which is mechanically stable in the whole range of $H$.

$\left(2 r / H_{0}=10\right)$, cut-off radius $\left(r_{0}=0.3 \mathrm{~nm}\right)$, and Poisson's ratio $(0.3)$, we obtain $k=5 \mathrm{GPa} / \mathrm{nm}$. The condition $\partial p(H) / \partial H>k$ leads to a hysteresis region for $H$ in the range $0.1 \mathrm{~nm}<H<0.6 \mathrm{~nm}$. At lower values of Young's modulus ( $E=20 \mathrm{GPa}$ ) the hysteresis region is slightly broader. Curve (b) in Figure 4 shows the function $p(H)$ for a stable pore, if the stability condition $\partial p(H) / \partial H<k$ holds in the whole range of $H$. A pore of this type could continuously close and reopen without any hysteresis by varying the condensate tension $p(H)$.

\section{Summary and Conclusions}

Sorption hysteresis at moderate and low humidity, which is often observed in cement based materials, could be explained by strong cohesion forces which attract the walls of narrow slit pores. The theoretical model presented here suggests a possible mechanism that can lead to a sorption hysteresis loop accompanied with a related loop of the pore volume change. Evaluating the elastic energy of closed slit pores, the attraction force between pore walls was found to be sufficiently large to close narrow slit pores. Monte-Carlo simulations and experimental results suggest that the attraction force between pore walls is substantially stronger than the conventional capillary tension predicted by the macroscopic theory of capillarity [8]. This strong attraction originates from divalent cations such as calcium ions, which are solved in the condensed water. The surface energy attributed to the adhesion between two solid interfaces can stabilize the closed configuration of narrow slit pores.

According to the elasticity theory, closing and reopening of slit pores depends on the Young modulus $E$, the slit thickness $H_{0}$, the aspect ratio $2 r / H_{0}$ of the pore and the adhesion energy $w$ of the solid surfaces. If a parameter $K \propto E H_{0}^{2} / w r$ is smaller than a certain threshold value, a closed slit pore remains permanently sealed. However, if the value of $K$ exceeds this threshold, a closed slit pore can reopen after reducing the magnitude of the scaled condensate tension $\Pi=p / p_{0}$. In the case of broad slit pores the tension $\Pi \propto \ln (m)$ can be controlled by changing the relative humidity $m$ (Equation (12)). In narrow slits the dependence of the tension $\Pi$ on the relative humidity $m$ is weaker than that in broad slits [8].

However, apart from changing $\Pi$, a closed slit pore can also reopen when the stiffness of the solid skeleton is increasing. Actually, the modulus of elasticity at the saturation point of water vapor $(m=1)$ was found to be about twice as high as its value at $m=0.2$. Thus, micropores can reopen when the stiffness of the solid skeleton increases with increasing relative humidity. Hardened cement pastes have a relatively large Young modulus. We expect that only narrow slit pores of these rigid materials can deform remarkable and produce hysteresis loops at low humidity. According to the microscopic description of the condensate-induced pore compression, a necessary condition for hysteresis is that the condensate tension $p(H)$ varies and crosses stability limits $H_{1}$ and $H_{2}$ (Figure 4), where the equation $\partial p(H) / \partial H=k$ holds. The elucidation of the hysteresis behavior requires more detailed knowledge about the dependence of $p(H)$ on the relative humidity $m$. Our theoretical approach is focused on narrow slit pores embedded in soft and moderately rigid solids. Narrow slit pores are supposed to occur in cement-based materials. For example, the Feldman-Sereda model for hardened cement pastes postulates narrow slit-like pores filled with an aqueous condensate. Jenning's model of cement pastes could lead to similar results on sorption hysteresis if physically bound water in globules is considered as a condensate in narrow slit pores. We suggest that in this case the definition of the material parameter $k$ (Equation (6)) should be replaced 
by $k=E / L[11]$, where $L$ is the diameter of a globule.

It should be noted, however, that there are also other attempts to explain low-humidity sorption hysteresis without changing the pore volume in cement pastes [15] [16]. It would be interesting to check experimentally whether wall distances of narrow slit pores in hardened cement pastes are changing by varying the humidity. Such a check could be possible by using the small angle neutron scattering method described by Chiang et al. [1].

\section{Acknowledgements}

Financial support by the German Research Foundation DFG (grant MO 600/9-1) is gratefully acknowledged.

\section{References}

[1] Chiang, W.-S., Fratini, E., Baglioni, P., Liu, D. and Chen, S.-H. (2012) Microstructure Determination of Calcium-Silicate-Hydrate Globules by Small-Angle Neutron Scattering. The Journal of Physical Chemistry C, 116, 5055-5061. http://dx.doi.org/10.1021/jp300745g

[2] McDonald, P.J., Rodin, V. and Valori, A. (2010) Characterisation of Intra- And Inter-C-S-H Gel Pore Water in White Cement Based on an Analysis of NMR Signal Amplitudes as a Function of Water Content. Cement and Concrete Research, 40, 1656-1663. http://dx.doi.org/10.1016/j.cemconres.2010.08.003

[3] Gelb, L.D., Gubbins, K.E., Radhakrishnan, R. and Sliwinska-Bartkowiak, M. (1999) Phase Separation in Confined Systems. Reports on Progress in Physics, 62, 1573-1659. http://dx.doi.org/10.1088/0034-4885/62/12/201

[4] Ippei, M., Igarashi, G. and Nishioka, Y. (2015) Bimodal Behavior of C-S-H Interpreted from Short-Term Length Change and Water Vapor Sorption Isotherms of Hardened Cement Paste. Cement and Concrete Research, 73, 158-168. http://dx.doi.org/10.1016/j.cemconres.2015.03.010

[5] Feldman, R.F. and Sereda, P.J. (1968) A Model for Hydrated Portland Cement Paste as Deduced from Sorption-Length Change. Materiaux et Constructions, 1, 509-520. http://dx.doi.org/10.1007/BF02473639

[6] Jennings, H.M. (2000) A Model for the Microstructure of Calcium Silicate Hydrate in Cement Paste. Cement and Concrete Research, 30, 101-116. http://dx.doi.org/10.1016/S0008-8846(99)00209-4

[7] Jönsson, B., Nonat, A., Labbez, C., Cabane, B. and Wennerström, H. (2005) Controlling the Cohesion of Cement Paste. Langmuir, 21, 9211-9221. http://dx.doi.org/10.1021/la051048z

[8] Bonnaud, P.A., Ji, Q., Coasne, B., Pellenq, R.J.-M. and Van Vliet, K.J. ( 2012) Thermodynamics of Water Confined in Porous Calcium-Silicate Hydrates. Langmuir, 28, 11422-11432. http://dx.doi.org/10.1021/la301738p

[9] Barrat, J.-L. and Hansen, J.-P. (2003) Basic Concepts for Simple and Complex Liquids. Cambridge University Press, Cambridge. http://dx.doi.org/10.1017/CBO9780511606533

[10] Beaudoin, J.J., Raki, L., Alizadeh, R. and Mitchell, L. (2010) Dimensional Change and Elastic Behavior of Layered Silicates and Portland Cement Paste. Cement \& Concrete Composites, 32, 25-33. http://dx.doi.org/10.1016/j.cemconcomp.2009.09.004

[11] Schiller, P., Wahab, M., Bier, Th., Waida, S. and Mögel, H.-J. (2015) Capillary Forces and Sorption Hysteresis of Cement Pastes with Small Slit Pores. Proceedings of the 5th International Biennial Conference on Ultrafine Grained and Nanostructured Materials, UFGNSM15. Procedia Materials Science, 11, 649-654. http://dx.doi.org/10.1016/j.mspro.2015.11.010 http://www.sciencedirect.com/science/article/pii/S2211812815003521

[12] Landau, L.D. and Lifschitz, E.M. (1986) Theory of Elasticity. Elsevier Butterworth-Heinemann, Oxford.

[13] Hull, D. and Bacon, D.J. (2011) Introduction to Dislocations. Elsevier, Amsterdam.

[14] Wan, K.-T., Smith, D.T. and Lawn, B.R. (1992) Fracture and Contact Adhesion Energies of Mica-Mica, Silica-Silica, and Mica-Silica Interfaces in Dry and Moist Atmospheres. Journal of the American Ceramic Society, 75, 667-676. http://dx.doi.org/10.1111/j.1151-2916.1992.tb07857.x

[15] Bazant, Z.P. and Bazant, M.Z. (2012) Theory of Sorption Hysteresis in Nanoporous Solids: Part I Snap-Through Instabilities. Journal of the Mechanics and Physics of Solids, 60, 1644-1659. http://dx.doi.org/10.1016/j.jmps.2012.04.014

[16] Bazant, Z.P. and Bazant, M.Z. (2012) Theory of Sorption Hysteresis in Nanoporous Solids: Part II Molecular Condensation. Journal of the Mechanics and Physics of Solids, 60, 1660-1675. http://dx.doi.org/10.1016/j.jmps.2012.04.015 